\title{
BMJ Open Analysis of medical services provided to patients with ankle sprains in Korea between 2015 and 2017: a cross-sectional study of the health insurance review and assessment service national patient sample database
}

\author{
Ho-sun Ryu, ${ }^{1}$ Boyoung Jung (10, 2 Jiyoon Yeo, ${ }^{3}$ Jae-Hong Kim, ${ }^{4}$ Dongwoo Nam, ${ }^{5}$ \\ In-Hyuk $\mathrm{Ha}$ (i) ${ }^{6}$
}

To cite: Ryu H, Jung B, Yeo J, et al. Analysis of medical services provided to patients with ankle sprains in Korea between 2015 and 2017: a cross-sectional study of the health insurance review and assessment service national patient sample database. BMJ Open 2020;10:e039297. doi:10.1136/ bmjopen-2020-039297

- Prepublication history and additional materials for this paper is available online. To view these files, please visit the journal online (http://dx.doi. org/10.1136/bmjopen-2020039297).

Received 23 April 2020

Revised 11 August 2020

Accepted 20 August 2020

A Check for updates

(C) Author(s) (or their employer(s)) 2020. Re-use permitted under CC BY-NC. No commercial re-use. See rights and permissions. Published by BMJ.

For numbered affiliations see end of article.

Correspondence to

Dr In-Hyuk Ha;

hanihata@gmail.com

\section{ABSTRACT}

Objectives To provide useful information for policymakers and clinicians by analysing the medical service use- divided into Western medicine (WM) and Korean medicine $(\mathrm{KM})$ —of patients with ankle sprains in South Korea between 2015 and 2017.

Design Cross-sectional, retrospective, observational study.

Setting Tertiary hospitals, WM hospitals, WM clinics, KM hospitals, KM clinics and others in South Korea.

Participants We analysed claim data and patient information from the 2015 to 2017 Health Insurance Review and Assessment National Patient Sample (HIRA-NPS) dataset, including 151415 patients diagnosed with a 'dislocation, sprain and strain of joints and ligaments at ankle and foot level' (10th revision of the International Statistical Classification of Diseases code S93) who used medical services at least once in 3 years between January 2015 and December 2017 in South Korea.

Primary and secondary outcome measures Cost of medical care, number of consultations, type of institution visited, types of treatment.

Results There were 160200 consultations and 53 044 patients in 2015, 149956 consultations and 50 830 patients in 2016 and 140651 consultations and 47541 patients in 2017. The total treatment costs were US\$3 355044.21 , US\$3 245827.70 and US\$3 128938.46 in 2015, 2016 and 2017, respectively. The most common age was 10-19 years. The most frequent type of visit was KM outpatient visit (56\%). Physiotherapy was most common in WM outpatient visits, while acupuncture was most common in $\mathrm{KM}$ visits. Most patients used one institution, rather than alternating between WM and KM.

Conclusions By identifying the trends and costs of treatment methods used for ankle sprains and comparing WM and KM, our data provide basic information for future health policy-making. In addition, the duality of the Korean medical system is highlighted as a possible cause of increased costs.
Strengths and limitations of this study

- The first strength of this study is that it is the first national-level analysis of medical service use of patients with ankle sprains over 3 years (2015-2017) in Korea.

- The second strength is that it provided a detailed analysis of the medical care supplied under the dialysed Korean healthcare system that includes Western medicine and Korean medicine.

- The third strength is that it provided information about the process through which patients with ankle sprains visit the medical institutions in practice by analysing institution choice.

Due to diagnostic accuracy of claim data, our results may be different if the main diagnosis is narrowed or if patients diagnosed with ankle sprain as the secondary diagnosis are included.

- The limitation is that we only analysed costs that occurred as a result of medical services and did not include non-medical costs (including transit costs for visiting the medical institution to receive medical care and costs associated with caregiving).

\section{INTRODUCTION}

Ankle sprains involve soft tissue damage as a result of excessive inner, outer and rotational movement of the ankle joint, leading to damage to the tendon complex of the ankle. Most minor ankle sprains recover completely with timely treatment. However, 10\%-30\% of ankle sprains progress to chronic ankle sprains, which show continuous pain and repeated symptoms of sprains. ${ }^{1}$ In addition, illnesses that are comorbid with ankle sprains (eg, injury to the peroneal tendon, fracture to the bone cartilage of the ankle joint or synovial injury, injury to the inner ligament, injury to the guard ligaments and traction injury to 
the nerves near the ankle) can cause chronic pain after sprains. ${ }^{2}$ As shown, ankle sprains occur frequently both in South Korea and overseas, and relapse is common. ${ }^{2}$

Ankle sprains occur in 2.15 out of 1000 people on average per year in the USA. ${ }^{3}$ Furthermore, ankle sprains occur most often in young people (15-19 years) in the USA. In terms of sex, it is reported that ankle sprains most commonly occur in men aged 15-19 years and in women aged 30 years or older. ${ }^{3}$ According to the National Health Insurance (NHI) Statistical Yearbook in South Korea in 2017, ${ }^{4}$ dislocation, sprain and strain of joints and ligaments at the ankle and foot level (10th revision of the International Statistical Classification of Diseases (ICD-10) code: S93) are associated with ankle sprain. The number of patients with ICD-10 code S93 was 1878 670. There were 898317 men (ranked 30th) and 980353 women (ranked 39th) with S93. In particular, it was the fifth most common cause of traditional outpatient visits, and the number of outpatient patients in traditional medicine with ICD-10 code S93 was 1006652 .

According to the Centers for Disease Control and Prevention, the estimated lifetime medical costs for ankle dislocations or sprains in 2010 will be US\$2 441377000 (US\$4 199168440 in 2019), or US\$1535 per person (US\$1800 in 2019). The estimated lifetime cost of lost workplace productivity will be US\$2 062693000 (US\$2 418378326 in 2019), resulting in total estimated lifetime costs due to ankle sprains of US\$4 504069000 (US\$5 280 738747 in 2019).

The resulting medical expenses are substantial, amounting to $>$ US $\$ 250$ million per year, and the substantial medical costs of ankle sprains have also been highlighted in other research. ${ }^{5-8}$ Medical costs associated with ankle sprains in South Korea were about US $\$ 179.8$ million (S93) (US\$1=1170 South Korean won (KRW); May 2019). In terms of Korean medical care in 2017, for S93, there were 998066 patients and US $\$ 76.7$ million in consultation costs, which equals a per-patient cost of 243 302 KRW (US\$202.08).

The Korean medical system is characterised by the use of Western or Korean traditional medicine at the patient's choice. ${ }^{9}$ Medicine-based pain treatment and physical therapy are mainly used to treat ankle sprains in Western medicine (WM) clinics; acupuncture, moxibustion, cupping and herbal medicine treatments are mainly used in Korean traditional medicine clinics. ${ }^{10}$ There is pressure from the public for patients to use Korean traditional medicine, because Korea's health insurance coverage rate was set up based on Korean traditional medicine clinics, which have lower costs than WM clinics have. ${ }^{11}$

Previous studies have recommended treating ankle sprains with early guide-accompanied movement, weight loading and taping or support, as needed. ${ }^{12-15}$ However, ankle sprains are often considered likely to develop into chronic diseases if they are not treated in time. Conversely, even mild ankle sprains can become problematic due to excessive treatment. ${ }^{16}$ Therefore, it is important to identify the prevalence of ankle sprains by country ${ }^{17}$ and the treatment trends ${ }^{18}$ to provide appropriate guidelines and cost-effective treatment for ankle sprains. However, to date, large-scale studies of ankle sprains are not sufficient, so epidemiological study of the treatment type and cost of ankle sprains is needed.

This study analysed data from the Health Insurance Review and Assessment Service (HIRA) to compare and analyse the current rates, treatment trends and visit routes for ankle sprains. We divided treatment into both Korean medicine (KM) and WM to compare multiple aspects (frequency of treatment, treatment cost, treatment method, institution choice, etc) using the HIRANational Patient Sample (HIRA-NPS) database. Through this study, it is possible to provide basic utilisation data of ankle diseases to policy makers and to provide evidence to clinicians for standard clinical guidelines of ankle diseases. Lastly, the public's right to know is guaranteed by providing the patient with an analysis based on institution choice.

\section{METHODS}

\section{Data and subjects}

Unlike most countries in the world, Korea has a single health insurance claims data source that can represent the entire nation, and these resources can be used as basic data for research related to national policy establishment and public health promotion in the health and medical field. Health insurance claims data are generated when healthcare facilities make insurance benefit claims with the HIRA after providing medical services to patients. Claims data contain massive amounts of diverse information including details of care (eg, treatment, procedure, examination and prescription), patient demographics, information about care provider and cost. Cost was defined as the cost of care delivered to a health insurance beneficiary at a care facility. It is the sum of the NHI-covered cost and the patient's out-of-pocket cost, as determined by a review of the total cost of care claimed by a care facility. ${ }^{19}$ This information is highly valuable for medical research because it is a representative dataset that can represent the medical use history of all Koreans.

The Korea HIRA developed sample data of representative health insurance data in Korea as a way to meet the demands of various fields for health insurance claim data. The purpose of developing and providing sample data is to promote healthcare research by improving understanding and utilisation of health insurance data. The health insurance claim data, which is the target population of HIRA-NPS, is very large. Therefore, the HIRA provide sampling by the number of observations that can secure versatility and representativeness for the convenience of researchers.

The data for this study were obtained from the 2015, 2016 and 2017 HIRA-NPS datasets provided by HIRA. ${ }^{20}$

The HIRA provides annual sample data (HIRA-NPS) that has been extracted via random stratified sampling to provide researchers with convenient access to data. 
Since 2017 , the sampling rate has been $3 \%$ of all patients (approximately 1.45 million). These secondary data extracts exclude personally identifiable information and corporate information from the raw data, and they contain insurance claims for a 1-year period from the date of care initiation for the corresponding year. Patients who used medical services during a 1-year period were selected via systematic, stratified sampling by sex and age group (5-year units), and their details of care and prescriptions are provided. ${ }^{20}$

Three per cent of the database was sampled, leading to the inclusion of about 1.4 million patients in 2015 and 2016 and about 1.45 million patients in $2017 .^{19}$

\section{Ankle injury}

Analysis was conducted on 'dislocation, sprain and strain of joints and ligaments at ankle and foot level (S93)', which is the ICD-10 code for ankle sprains. This standard followed previous research that used the S93 ICD-10 code to analyse ankle sprains. ${ }^{21}$ Only patients with S93 in the primary diagnosis were included, and patients with other illnesses recorded in primary diagnosis and S93 in secondary diagnosis were excluded. Through this process, a total of 451070 claims without a secondary diagnosis were extracted out of 794752 claims in the HIRA-NPS data between 2015 and 2017. Among those, cases where the total cost was $0 \mathrm{KRW}$ or cases where the date of visit was missing were excluded, resulting in a final sample of 450807 claims (151 415 patients) for analysis (figure 1).

\section{Data analysis}

The characteristics of the patients with ankle sprains were analysed by age, sex, public insurance scheme, hospital, medical specialty and medical institution. Age was categorised in 10-year increments, sex was categorised as male/ female and public insurance scheme was categorised as NHI or Medicaid. Medicaid is a system provided by the state to provide medical assistance to basic living recipients with low incomes. For Medicaid, an individual qualifies if the income of their household is <US\$600 per month. As of 2018, 52556653 patients were covered by NHI in Korea, and 1484671 patients were covered by

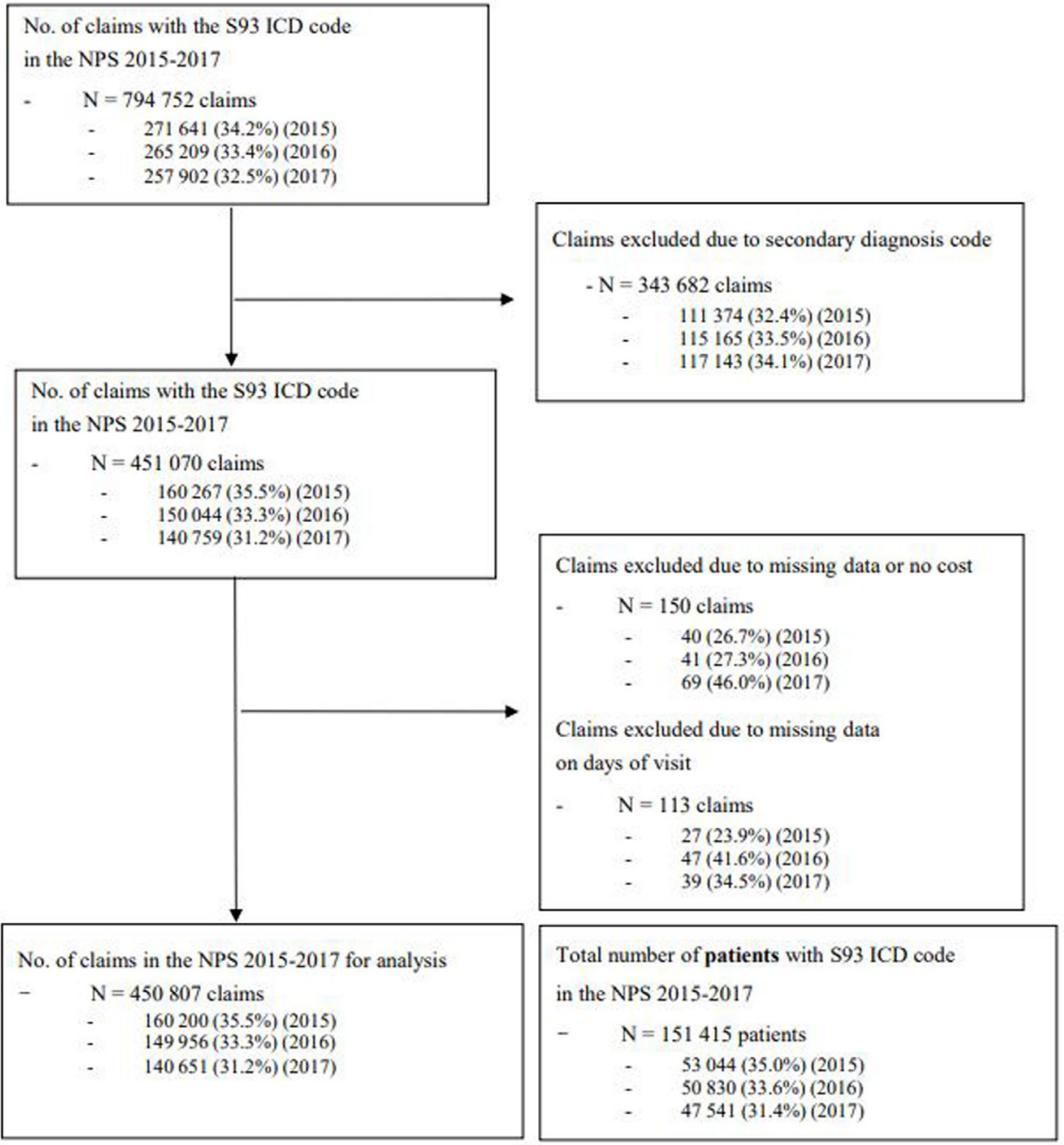

Figure 1 Flow chart of the study of inclusion and exclusion of participants from the 2015-2017 Health Insurance Review and Assessment National Patient Sample (HIRA-NPS). Ankle sprain; S93 10th revision of the International Statistical Classification of Diseases code. 
Medicaid. Medical institutions were classified as one of the following: tertiary and general hospitals, hospitals, western clinics, Korean traditional hospitals and Korean traditional clinics. The division of WM and $\mathrm{KM}$ was based on medical institutions. The categories belonging to KM include traditional hospitals and traditional clinics; others are divided into WM.

In accordance with the recommendations of the Centers for Disease Control and Prevention (https:// wisqars.cdc.gov: $8443 / \operatorname{costT} /$ ), total costs were analysed based on the following categories made by the Ministry of Health and Welfare: consultation fee, hospitalisation, medication, injection, anaesthesia, physiotherapy, procedure and surgery, examination, imaging and radiography. The reason we did the analysis based on that criterion was that we were based on the 10 classification criteria of claims data.

Medical costs determined to be eligible for reimbursement by HIRA out of treatment costs were indicated in the submitted insurance claim statement. Medical costs are the sum of benefits reimbursed by the insurer (Korean NHI Service) to the medical care institutions and self-payment costs paid by the beneficiary (patient). The total expense was evaluated, approved and deducted by the NHI out of the total cost billed by the institution. Per-patient expense and per-case expense was calculated as the sum of costs listed on the number of patient and their claims, respectively.

Surgeries, injections, physiotherapy and use of pain killers were analysed. Frequency analysis was performed based on the number of cases of ankle sprains who were given a code and the bills. Here, the surgery code did not exist independently and was always tied to the procedure and surgery items. Therefore, codes related to treatment were excluded from the code for procedure and surgery. Surgeries that were not limited to the ankles were excluded because of the possibility that surgeries for another part of the body were included.

The treatment codes for ankle sprains were categorised separately in the order of higher frequency for KM and WM. WM outpatient care, WM inpatient care, KM outpatient care and KM inpatient care only included those that were billed at least 200 times, 50 times, 1500 times and 50 times in 3 years, respectively. The number of procedures below these numbers was very small compared with the number of examinations, and the frequency was not constant when comparing 3 years. Therefore, it was regarded as irrelevant to normal treatment of ankle sprains and was excluded from the study population. As a result, the top 14 cases of WM outpatient care, top 13 cases of WM inpatient care, top 13 cases of KM outpatient care and top 7 cases of KM inpatient care were analysed. The prescribed medicine was categorised by the frequency of prescription for ankle sprains, by converting the main ingredient code applied during inpatient and outpatient visits to the Ministry of Health and Welfare categories. If a drug accounted for $<3 \%$ in all 3 years, it was excluded from the analyses. Because these drugs did not show a constant frequency in each year, they were not considered as drugs related to the treatment of conventional ankle sprains and were excluded. (These drugs are documented in online supplemental table 1 . Total dosage of drug prescriptions for ankle sprains.)

In addition, patient institution choice was classified into six groups (groups 1-6). (Institution choices were excluded if their frequency was 10 times or less.) Frequency, percentage, mean, SD and rate of change were provided for each value. All statistical analyses were performed using SAS V.9.4 TS Level 1M1 (2002-2012 by SAS Institute, Cary, North Carolina, USA).

\section{RESULTS}

Number of patients and cost of medical care for ankle sprains Patients diagnosed with injury S93 of their ankles were sampled from the 2015, 2016 and 2017 HIRA-NPS. There were 271641 consultations in 2015, 265209 consultations in 2016 and 257902 consultations in 2017. Among those, patients whose secondary diagnosis was S93, patients whose total cost was $0 \mathrm{KRW}$ and did not receive treatment and patients with missing data were excluded. In the final sample, there were 160200 consultations and 53044 patients in 2015, 149956 consultations and 50 830 patients in 2016 and 140651 consultations and 47 541 patients in 2017 (figure 1). Overall, the number of patients was decreasing, resulting in a decrease in the number of consultations as well.

Total medical costs were US\$3 355044.21 , US\$3 245 827.70 and US\$3 128938.46 , US $\$$, in 2015, 2016, and 2017, respectively, showing a decreasing trend. However, the cost per patient and the cost of consultation increased.

In cases of ankle sprains who received WM outpatient care, the number of visits to the clinic during the duration of the treatment was about three. For ankle sprains that required hospitalisation, the number of days of care was about 10 days on average (table 1 ).

\section{Characteristics of patients with ankle sprains and medical use}

The frequency of the illness by age group showed similar trends across the 3 years. The highest frequency was observed in the 10-19 years age group, exceeding $20 \%$ in all 3 years (mean $21.44 \%$ ). The $20-59$ years age groups showed similar proportions, followed by a rapid decrease from the age of 60 . The number of visits to the clinic showed an increasing trend with increasing age. Patients in the $0-9$ years age group had $<2.5$ visits, while patients who were 70 years or older had 4 or more visits. Overall, there were more female than male patients $(53.9 \%$ vs $46.1 \%)$. The number of visits per patient was higher for women than for men.

More than $97 \%$ of the patients were insured with NHI. Generally, the number of visits was slightly higher for patients insured with Medicaid than for patients insured with NHI. Surgeries were rare, and they were performed 


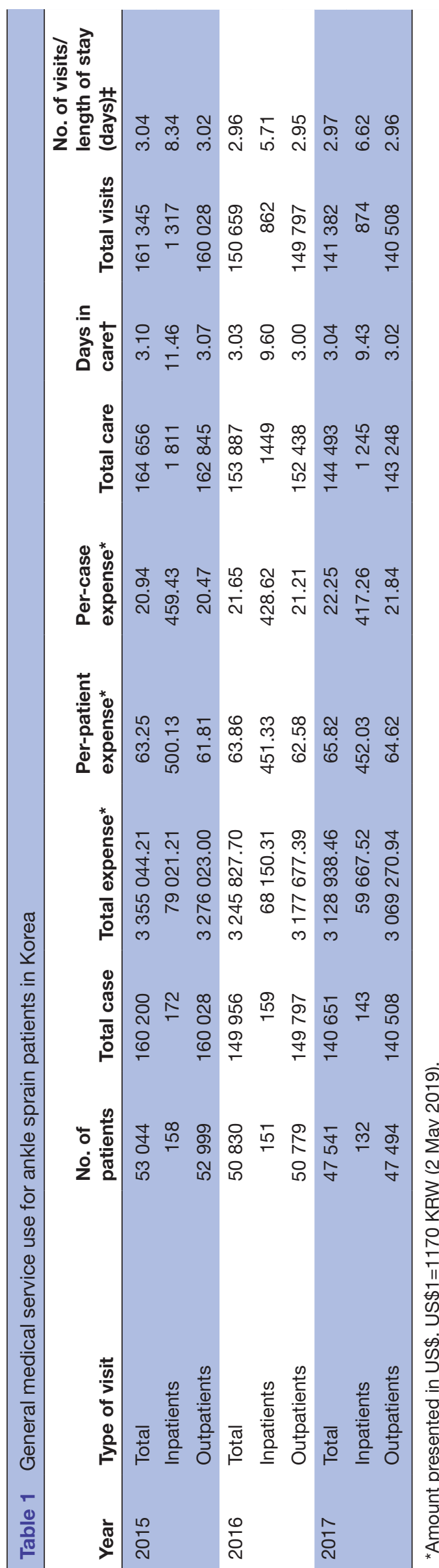

in $<0.1 \%$ of all cases. If a surgery was performed, the number of visits increased by a factor of about 2-3.

The most frequently visited medical institutions were KM outpatient institutions (about $56 \%$ ), followed by WM outpatient institutions (about 51\%). The percentage here includes cases in which one patient has visited other medical institutions, so the total may exceed $100 \%$. Hospitalisations were rare. For KM outpatient visits, the mean number of visits was 3.7 visits, which was higher than the mean number of visits for WM outpatient visits (2.4 visits). By medical specialty, patient frequency was the highest in the order of orthopaedic surgery (OS), internal Korean medicine, Department of Acupuncture and Moxibustion Medicine and general surgery (GS).

More than $90 \%$ of all care occurred at the clinic level (WM clinic: $39.49 \%$; KM clinic: $54.82 \%$ ), which are primary medical institutions. There were no significant differences between the frequencies of visits to the hospital-level and clinic-level institutions (table 2).

\section{Medical costs of ankle sprains by patient characteristics}

The results of analysing the total bill by age groups showed that the cost was the greatest for the 10-19 years age group, who frequently experience ankle sprains, and that it rapidly decreased after 60 years of age. The cost per day showed a gradual decreasing trend with increasing age. The cost per visit was higher for men than for women, and higher for WM outpatient visits than for KM outpatient visits. For the medical specialty of WM, the cost per visit was more than four times higher for emergency medicine (EM) visits compared with other specialties. Consultation cost per visit increased with increasing level of medical institution (online supplemental table 2).

\section{Medical costs by the category of treatment}

The cost of consultation accounted for $>45 \%$ of the total medical cost every year: among only WM consultations, the most frequently billed cost was for consultation, comprising $>40 \%$ in all 3 years. While procedure and surgery accounted for $9 \%$ of all cases, its total cost was ranked the second highest at about $24 \%$. Both billing frequency and costs of radiography treatments were about $16 \%-17 \%$. While the frequency of physiotherapy was second highest at $24 \%$, the cost accounted for about $10 \%$ of the total.

For KM visits, consultations and acupunctures comprised similar proportions (about $48 \%$ ) of the total number of cases and comprised most of the cost with about $60 \%$ being for acupunctures and about $39 \%$ being for consultations (table 3).

\section{Care and prescription}

Online supplemental table 3 shows the data for high frequency care for WM and KM. During WM outpatient visits, physiotherapy methods such as deep and superficial heat therapy, transcutaneous electrical nerve stimulation (TENS) and interference current therapy (ICT), were the most common, followed by short leg sprint for sprains. 


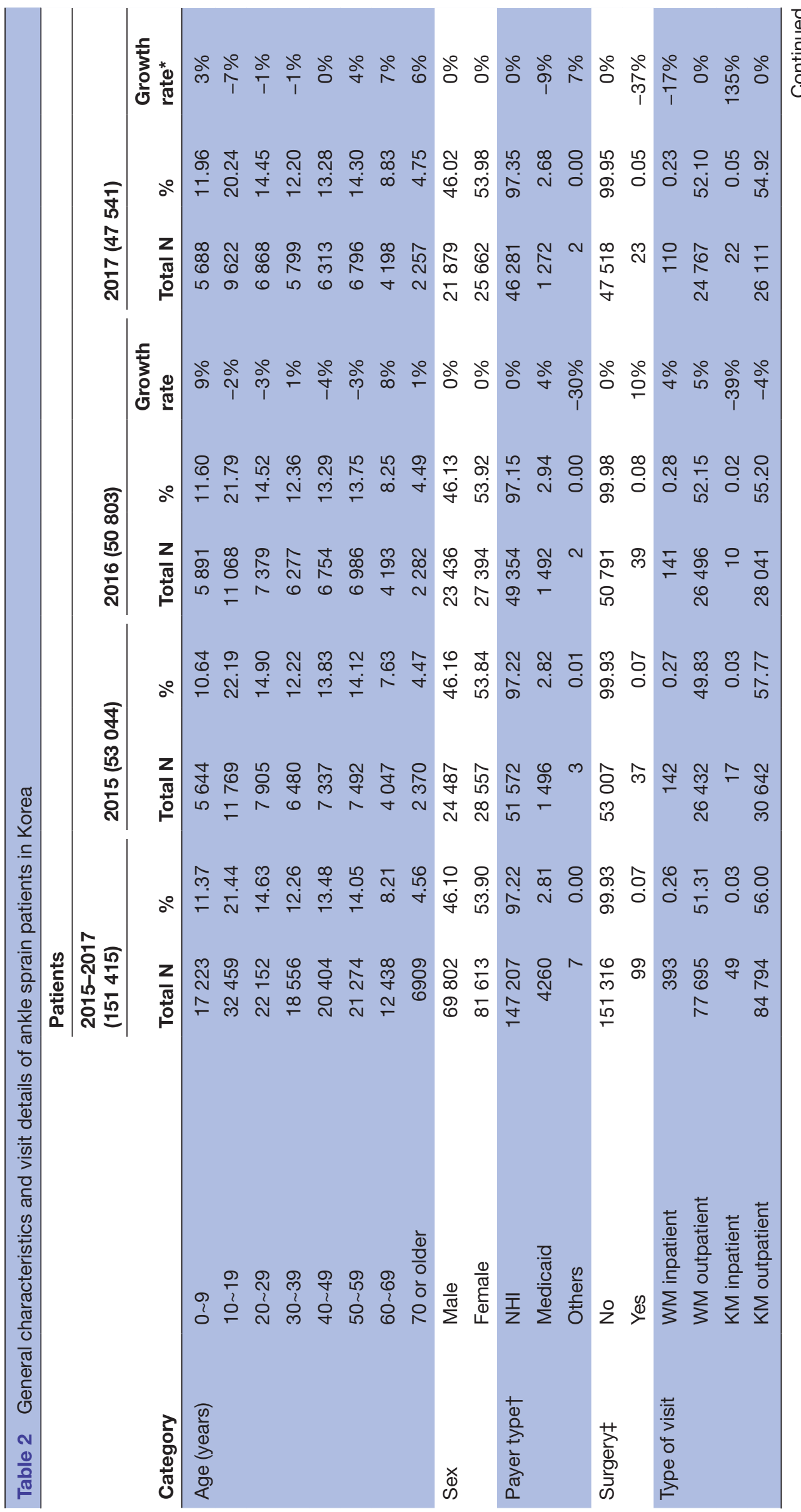



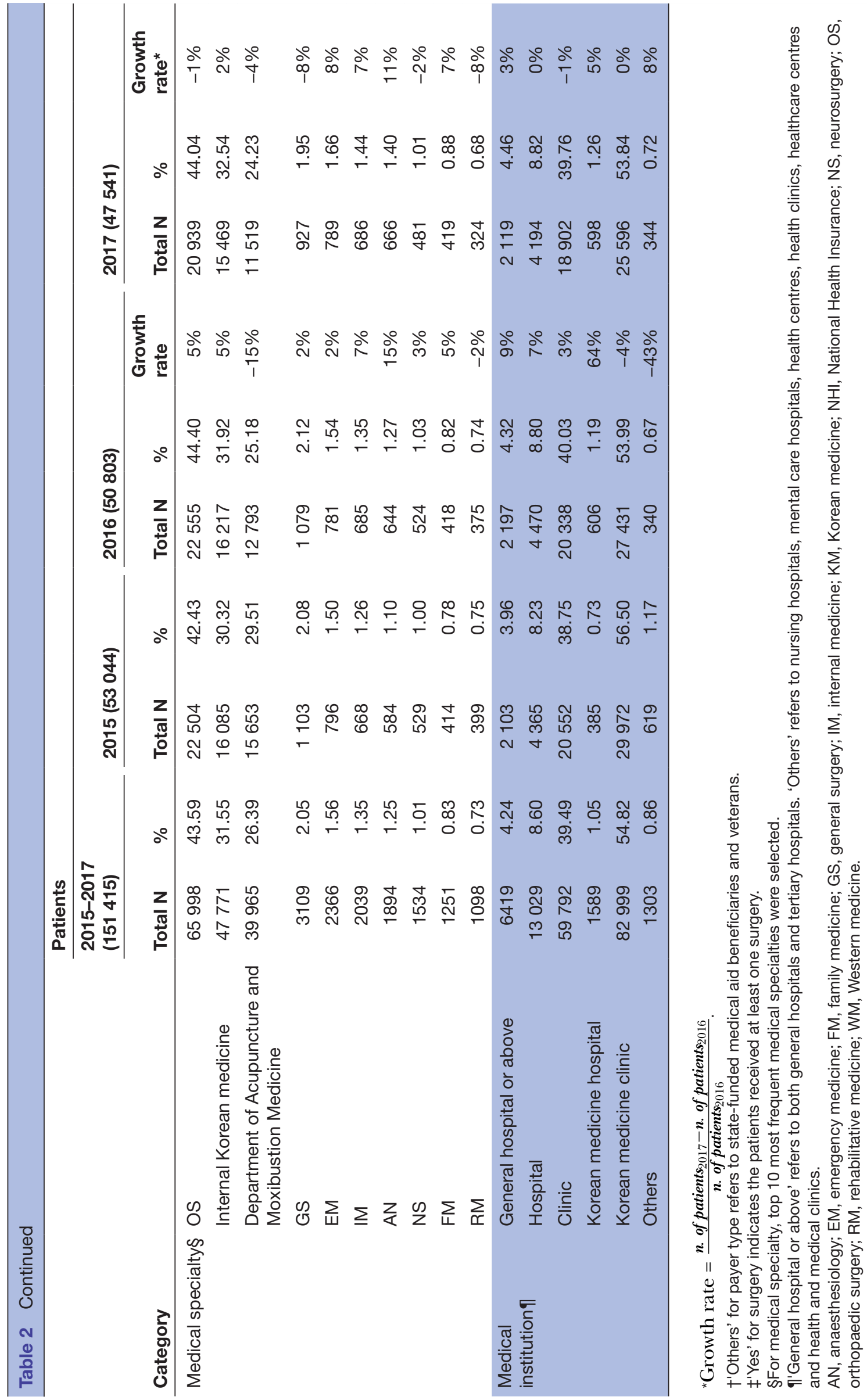


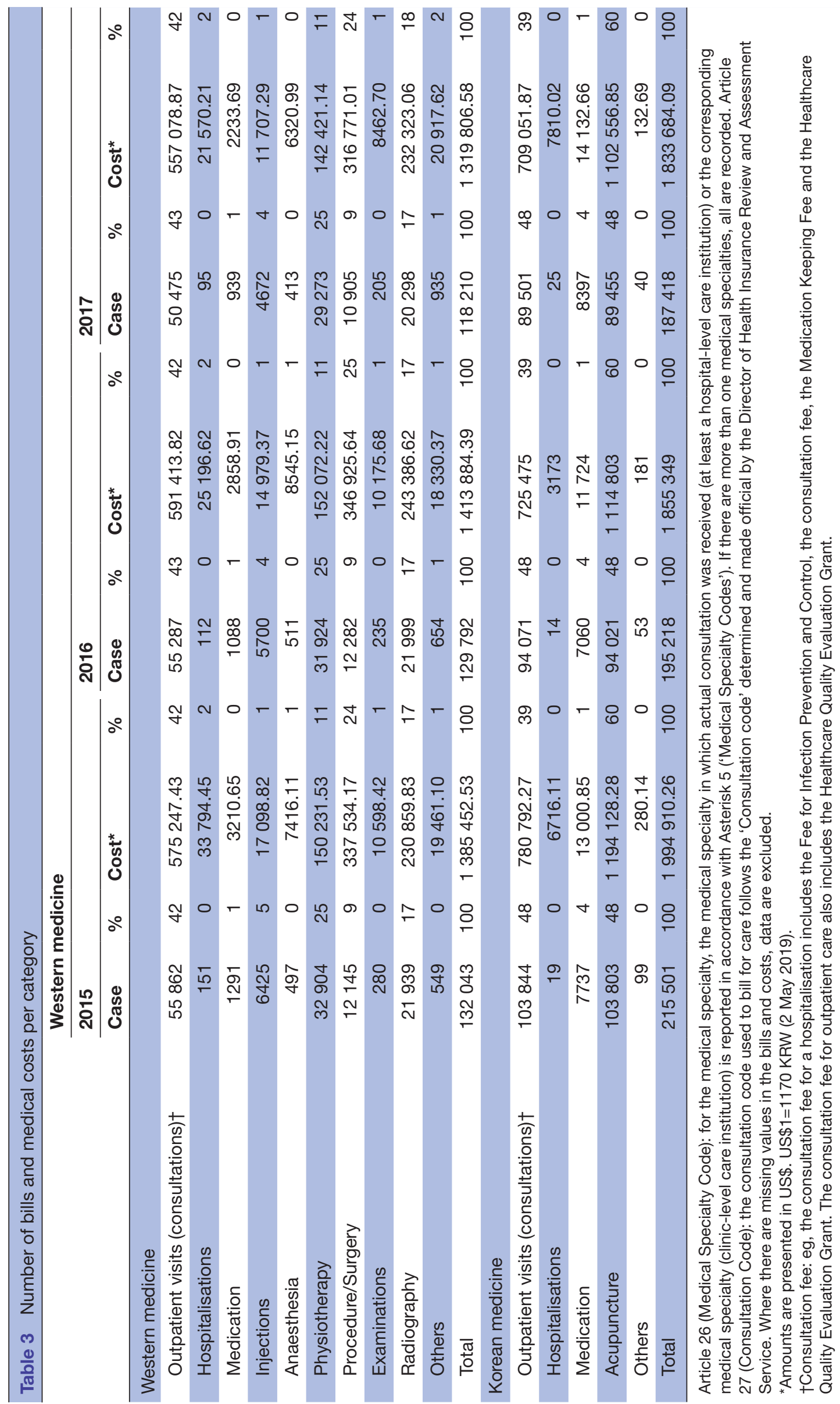


During WM inpatient visits, subcutaneous or intramuscular injections were performed often. The mean number of physiotherapy sessions performed during WM outpatient visits was similar to the mean number of visits (2.4). During KM inpatient and outpatient visits, acupuncture and cupping were the main methods used, followed by warming and cryotherapy of the meridian muscle and moxibustion.

The number of cases per item and the mean number of cases per patient were not proportional across WM outpatient visits and KM outpatient visits. Thus, it can be inferred that the same patient who visited medical institutions multiple times receives similar treatments (online supplemental table 3 ).

Online supplemental table 1 shows the total dosage of drug prescriptions made at WM outpatient and inpatient visits categorised by the Ministry of Health and Welfare categories. During WM outpatient visits, the total dosage of prescription of non-steroidal anti-inflammatory drugs (NSAIDs) accounted for $>45 \%$. Next, enzymes accounted for $>23 \%$, followed by gastrointestinal drugs. Furthermore, during WM inpatient visits, the total dosage of prescription of NSAIDs accounted for $>30 \%$ and the prescription of enzymes and gastrointestinal drugs exceeded $10 \%$. In 2017, the number of prescriptions of drugs for peptic ulcer were not counted due to the changes in Ministry of Health and Welfare categories. These drugs appear to have been counted as gastrointestinal drugs (online supplemental table 1).

\section{Visit route}

Investigation of medical institution visit routes showed that $>95 \%$ of the patients received care from either WM alone or KM alone, rather than alternating between the two. A total of $52.45 \%$ of the patients first sought KM care, and these patients were older than those who first sought WM care. In patients aged 60 years or older, $19.52 \%$ first sought KM and only $5.65 \%$ first sought WM. The group of patients who only received KM accounted for $50.78 \%$ of the total and were the oldest in age. The group of patients who only received WM accounted for $45.03 \%$ of the total and were the youngest in age (table 4 ).

Among those, group $3(0.16 \%)$, who received KM care followed by WM and back to KM care, was further analysed

\begin{tabular}{|c|c|c|c|c|c|c|c|c|c|}
\hline \multirow[b]{2}{*}{ Group } & \multirow[b]{2}{*}{ Age (years) } & \multicolumn{2}{|l|}{ Total } & \multicolumn{2}{|l|}{2015} & \multicolumn{2}{|l|}{2016} & \multicolumn{2}{|l|}{2017} \\
\hline & & $\mathbf{N}$ & $\%$ & $\mathbf{N}$ & $\%$ & $\mathbf{N}$ & $\%$ & $\mathbf{N}$ & $\%$ \\
\hline \multirow{4}{*}{$\begin{array}{l}\text { KM } \\
(50.9 \%)\end{array}$} & All & 72742 & 100.0 & 26218 & 100.0 & 23984 & 100.0 & 22540 & 100.0 \\
\hline & $<40$ & 30250 & 41.6 & 11244 & 42.9 & 10011 & 41.7 & 8995 & 39.9 \\
\hline & $40-59$ & 28295 & 38.9 & 10227 & 39.0 & 9274 & 38.7 & 8794 & 39.0 \\
\hline & $60+$ & 14197 & 19.5 & 4747 & 18.1 & 4699 & 19.6 & 4751 & 21.1 \\
\hline \multirow{4}{*}{$\begin{array}{l}\text { KM-WM } \\
(1.5 \%)\end{array}$} & All & 2172 & 100.0 & 795 & 100.0 & 748 & 100.0 & 629 & 100.0 \\
\hline & $<40$ & 1321 & 60.8 & 490 & 61.6 & 458 & 61.2 & 373 & 59.3 \\
\hline & $40-59$ & 645 & 29.7 & 243 & 30.6 & 216 & 28.9 & 186 & 29.6 \\
\hline & $60+$ & 206 & 9.5 & 62 & 7.8 & 74 & 9.9 & 70 & 11.1 \\
\hline \multirow{4}{*}{$\begin{array}{l}\text { KM-WM-KM } \\
(0.2 \%)\end{array}$} & All & 239 & 100.0 & 83 & 100.0 & 60 & 100.0 & 96 & 100.0 \\
\hline & $<40$ & 142 & 59.4 & 50 & 60.2 & 33 & 55.0 & 59 & 61.5 \\
\hline & $40-59$ & 77 & 32.2 & 29 & 34.9 & 20 & 33.3 & 28 & 29.2 \\
\hline & $60+$ & 20 & 8.4 & 4 & 4.8 & 7 & 11.7 & 9 & 9.4 \\
\hline \multirow{4}{*}{$\begin{array}{l}\text { WM } \\
(45.0 \%)\end{array}$} & All & 64517 & 100.0 & 21738 & 100.0 & 22140 & 100.0 & 20639 & 100.0 \\
\hline & $<40$ & 51477 & 79.8 & 17409 & 80.1 & 17706 & 80.0 & 16362 & 79.3 \\
\hline & $40-59$ & 9396 & 14.6 & 3171 & 14.6 & 3153 & 14.2 & 3072 & 14.9 \\
\hline & $60+$ & 3644 & 5.6 & 1158 & 5.3 & 1281 & 5.8 & 1205 & 5.8 \\
\hline \multirow{4}{*}{$\begin{array}{l}\text { WM-KM } \\
(2.4 \%)\end{array}$} & All & 3459 & 100.0 & 1330 & 100.0 & 1132 & 100.0 & 997 & 100.0 \\
\hline & $<40$ & 2358 & 68.2 & 934 & 70.2 & 771 & 68.1 & 653 & 65.5 \\
\hline & $40-59$ & 865 & 25.0 & 306 & 23.0 & 288 & 25.4 & 271 & 27.2 \\
\hline & $60+$ & 236 & 6.8 & 90 & 6.8 & 73 & 6.4 & 73 & 7.3 \\
\hline \multirow{4}{*}{$\begin{array}{l}\text { WM-KM-WM } \\
(0.1 \%)\end{array}$} & All & 117 & 100.0 & 30 & 100.0 & 48 & 100.0 & 39 & 100.0 \\
\hline & $<40$ & 85 & 72.6 & 20 & 66.7 & 36 & 75.0 & 29 & 74.4 \\
\hline & $40-59$ & 27 & 23.1 & 9 & 30.0 & 10 & 20.8 & 8 & 20.5 \\
\hline & $60+$ & 5 & 4.3 & 1 & 3.3 & 2 & 4.2 & 2 & 5.1 \\
\hline
\end{tabular}

KM, Korean Medicine; WM, Western medicine. 
Table 5 Radiography and casting rate for group 3 patients in Western medicine hospitals

\begin{tabular}{lrrrrrrr}
\hline & & \multicolumn{2}{c}{ Radiography } & & \multicolumn{2}{c}{ Cast } \\
\cline { 3 - 4 } \cline { 7 - 8 } Year & All & N & $\%$ & & N & $\%$ \\
\hline 2015 & 83 & 55 & 66.26 & & 17 & 20.48 \\
2016 & 60 & 50 & 83.33 & & 8 & 13.33 \\
2017 & 96 & 70 & 72.91 & & 19 & 19.79 \\
Total & 239 & 175 & 73.22 & & 44 & 18.41 \\
\hline
\end{tabular}

in terms of which treatments they received in their WM visit. Across 3 years, $73.22 \%$ on average visited both for radiography and $18.41 \%$ received a cast (table 5 ).

Additional analyses were performed to test whether group 3 and group 6 revisited the same clinic when they returned to the Korean or Western medical institution. The results showed that $73 \%$ and $80 \%$ of the patients returned to the same Korean and Western medical institutions, respectively.

\section{DISCUSSION}

There were no large differences in the proportions of inpatient and outpatient visits across the 3 years. The average rate of hospitalisation was $<0.3 \%$, and both the number of inpatient and outpatient patients decreased in the 3 years with a decrease in the total number of patients. The comparison of the total number of patients and the total number of cases showed that $<10 \%$ of hospitalised patients were re-hospitalised. Although the total cost of ankle sprains decreased, the cost per patient generally showed an increasing trend. The number of days of consultation at a hospital was about 3 days for outpatient care and 9 days for inpatient care.

The fact that the proportion of patients aged between 10 and 19 was the highest at $21.44 \%$ on average is in line with the findings from previous studies. ${ }^{4}$ The frequency of ankle sprains decreased to below average after 60 years of age. The mean number of visits by age group showed a continuous increase with increasing age. This suggests that ankle sprains are more severe or require a longer duration of treatment with increasing age. In terms of sex, ankle sprains were slightly more common in women than men, and the mean number of visits was also higher for women. The fact that ankle sprains occur more commonly among women has been studied in previous research. ${ }^{22}$ Ankle sprains are more common in the younger generation, as the main cause is the loss of the foot. Women are especially likely to wear high-heeled shoes to increase their chances of getting injured.

The number of visits among patients covered under Medicaid may have been higher because these patients are older in age and they show a higher frequency of visits to medical institutions compared with patients covered under NHI. ${ }^{23}$ Surgeries with ankle sprains as the main ailment were rare, accounting for $<0.1 \%$ of all cases. This is in line with previous findings that there were no clear differences between functional treatment and surgical treatments, and that there was no clear evidence of the clinical usefulness of surgical treatments in treating acute lateral ankle sprains. ${ }^{24}$ However, in the case of chronic instability that shows positive on repeated pain, giving way and load test, surgery may be necessary. ${ }^{25}$

The comparison between KM and WM showed that more patients visited KM institutions. Because the mean number of visits per patient was higher for KM, there also was a higher number of bills for KM than for WM. For specialties under WM, OS was by far the most common at $>83 \%$, followed by GS, EM and internal medicine. Given its characteristics, EM had the lowest frequency of visit. Other specialties under WM did not show significant differences. Most consultations occurred in primary care, that is, the clinics and KM clinics. Compared with visitors to the KM clinic, visitors to the KM hospital were low in number. Comparatively, the number of visitors to the hospitals or tertiary hospitals instead of clinics was relatively high (means of $39.5 \%$ for clinics, $8.6 \%$ for hospitals and $4.2 \%$ for tertiary hospitals).

The proportion of visits to clinics and hospital-level institutions increased slightly across 3 years for both WM and KM. This was related to the medical characteristics of Korea (concentration of patients to large hospitals) in which patients prefer upper-level institutions, even for minor ailments that can be treated in primary medical institutions. ${ }^{26}$ The number of visits per year to the KM clinics was $>3.6$ visits for all 3 years, showing around one more visit compared with the clinic-level institutions. The number of visits was the lowest for inpatient care at tertiary hospitals.

The medical cost per visit decreased with increasing age according to the bills. This appears to be because, as the number of visits increases with increasing age, the fees for initial consultation and examinations that are billed at the first visit are not billed again for subsequent visits, thus decreasing the medical costs of return visits. ${ }^{27}$ For similar reasons, the cost per visit was higher for women, who also had a higher number of visits compared with men. The fact that the number of bills relative to the number of patients increased with increasing age can be attributed to the impact of the increasing number of visits.

The medical cost per category on the bill was analysed separately for WM and KM. As a result, the categories with the highest cost in WM were consultation, procedure and surgery, radiography and physiotherapy. Online supplemental table 2 shows that the rate of surgery for ankle sprains was low, and thus it seems that most of the costs for procedure and surgery were for casting. Because the costs of procedure and surgery were both relatively high compared with other services, the total cost was the highest after consultation fees. As physiotherapy is relatively low cost, the total cost was relatively low compared with the number of cases. The comparison of the 3 years showed that the number of visits was decreasing due to the overall decrease in the number of patients. While costs of medication and injections were also decreasing by 
large amounts, care categories that cost much of the total cost such as radiography, physiotherapy and procedure and surgery maintained their costs.

It can be inferred that the proportions of medication prescription or injection were decreasing and that the proportion of radiography, physiotherapy and casting were staying at a consistent level or increasing. Using NSAIDs or casting can reduce the pain due to sprains before complete recovery. However, $30 \%$ of patients have been reported to be injured again because patients tend to exercise or exercise before recovery. ${ }^{28}$ According to previous research, overuse of painkillers ${ }^{29}$ and steroid injections ${ }^{30}$ is not recommended. Therefore, the fact that the proportion of Western prescriptions and injections decreased over the 3 years is considered positive.

In KM, the number of claims for consultation decreased every year. With this, the cost of consultation and acupunctures also decreased continuously. The similar number of bills for injections and for consultation shows that most patients in KM received acupunctures. However, the cost of medication was the highest in 2017, and it can be inferred that the rate of prescription in insured KM has increased. ${ }^{31}$

Treatments administered in WM and KM are shown in online supplemental tables 2 and 3. In most WM outpatient visits, physiotherapy (eg, heat therapy, TENS and ICT) were performed and NSAIDs were prescribed for the purposes of pain control. However, the effect of physiotherapy for acute ankle sprains remains unclear to date. ${ }^{32}$ The use of short leg splints was also common. The frequency of subcutaneous or intramuscular injections was high for WM inpatient visits compared with outpatient visits. In the case of KM, the number of bills for consultation, injection and procedure were similar. Thus, it can be inferred that most common treatment was acupuncture, followed by warming meridian (infrared ray irradiation), cupping and electric acupuncture stimulation. Among these, acupuncture comprised the largest number, thus showing that most treatments involved acupuncture. Acupuncture for ankle sprains is as effective as other conventional treatments, and complications are rare. ${ }^{33}$ However, the quality of randomised controlled trial research on this topic is not high, and additional research is needed. A large number of cupping procedures were also performed. This may be related to previous research findings that showed that using acupuncture and cupping together on ankle sprains can be more effective than using acupuncture alone. ${ }^{34}$ Because there are differences in the total number of treatments and the mean number of treatments per patient, it can be assumed that patients who return after receiving one type of care receive a similar type of care.

Table 5 shows that the preference for KM treatment increases with increasing age. This finding is in line with the preference for KM service among Korean citizens. ${ }^{33}$ With increasing age, the frequency of visiting a hospital due to ankle sprains increased further. Furthermore, it was found that higher frequency of visiting a KM institution was also related to the age of the patient. In other words, older age was associated with prioritised visits to KM clinics for ankle sprains. Therefore, the overall number of visits to the KM institutions was increased. Considering that only $5 \%$ of patients receive treatment in both WM institutions and KM institutions, the preference for medical institution for ankle ailments was distinct for each individual in Korea.

Online supplemental table 4 shows that a high proportion $(73 \%)$ of patients visited a KM institution, then a WM institution, followed by the same KM institution. It can be inferred that the visit to a different medical institution was because of the need for a medical service that cannot be fulfilled at the original institution given the differences in medical care in the WM institutions and the KM institutions. For this reason, higher numbers of radiography and casts were performed on patients who visited a WM institution after visiting a KM institution (table 5). This finding exemplifies the discomfort experienced by $\mathrm{KM}$ visitors who must visit a WM institution again for radiography to identify the fracture and skeletal shape. In Korea, western and traditional medicine are divided, and Korean medical doctors are unable to use imaging equipment such as X-rays or MRI. In the previous study, $20.8 \%$ of overlapping visits to traditional medicine were mentioned, and the increase in medical expenses was mentioned. ${ }^{35}$ Although the total number of these cases was not high in this study, it is assumed that these medical limitations cause time, effort, and discomfort for patients and will induce an overall increase in medical costs when the entire population of Korea is considered rather than the sample used in this study.

\section{Strengths and limitations}

First, there may have been errors in the accuracy of the data because patients were sampled based on the main diagnosis indicated by the claim data. ${ }^{19}$ Ankle sprain disease is classified only by ICD-10 code S93, so it is not possible to distinguish the grade of the ankle sprain (grades 1 and 2). According to previous research, the diagnostic accuracy of the bills submitted to the NHI is about $70 \%{ }^{36}$ To overcome this limitation, we only included subjects whose main diagnosis was a 'dislocation, sprain and strain of joints and ligaments at ankle and foot level (S93)'. The reason that we widened the ankle sprain category with S93 rather than S93.4 is that there may be errors in the data accuracy of the major diagnosis shown in the claim data. Although our study population has been defined broadly, it can show the characteristics of ankle sprain at the category level, because S93.6 and S93.4 account for $>90 \%$ of cases (online supplemental table 5). The number of patients who visited with ankle sprains as their main diagnosis decreased slightly from 2015 to 2017 . The results may be different if the main diagnosis is narrowed as 93.4 or if patients diagnosed with ankle sprain as the secondary diagnosis are included.

Second, medical costs are generally categorised into direct costs, indirect costs and intangible costs. ${ }^{37}$ However, 
this study only analysed direct costs that occurred as a result of medical services, while also excluding nonmedical costs (including transit costs for visiting the medical institution to receive medical care and costs associated with caregiving). There are a total of $201 \mathrm{KM}$ insurable items included in the Billing Data in South Korea. Compared with the 5611 insurable items in WM, this is a low number and the proportion of non-insured items is high. Therefore, it is difficult to conclude that the data billed by Korean medical institutions accurately reflect all KM treatments for patients with ankle sprains.

Despite these limitations, there were several strengths to this study. First, this study analysed the medical service use of patients with ankle sprains over 3 years of data from 2015 to 2017 in the HIRA-NPS. To date, there has been no study comparing the medical use of patients with ankle sprains by year at a national level. Second, we have described the medical care provided in WM and KM in terms of the special characteristics of Korea, which is a dualised healthcare system. Finally, this study provides information about the process through which patients with ankle sprains visit the medical institutions in practice by analysing their visit routes.

The results of the study are summarised as follows: patients with ankle sprains are predominantly young, with the incidence highest in teens; on average, patients visit medical institutions about three times a year; and patients mostly visit medical clinics. In particular, our study found that the cost of medication and injections/ procedures decreases and the cost of physical therapy or braces increases in patients who visited KM.

\section{CONCLUSION}

This study analysed the patient sample data over 3 years through the Billing Data from the HIRA service to examine the current state of medical service use for ankle sprains in South Korea. By identifying high frequency treatments and high costs associated with ankle sprains, this study provides information that is useful for insurance systems and health policy-makers to plan future directions. In addition, analysing the visits separately for $\mathrm{WM}$ and $\mathrm{KM}$ led to the inference that the dualised system caused an additional increase in cost, and it was confirmed that more patients visited $\mathrm{KM}$ institutions for ankle sprains. Considering that research comparing patients with ankle sprains has not yet been presented, it is anticipated that the findings of this study can be used as basic data for national health policy-making in the future, such as rational decision-making for appropriate care of patients with ankle sprains, determining the number of health insurance-covered services for related services, and determining funding.

\footnotetext{
Author affiliations

${ }^{1}$ Jaseng Hospital of Korean Medicine, Gangnam-gu, Republic of Korea

${ }^{2}$ Department of Health Administration, Hanyang Women's University, Seoul, Republic of Korea
}

${ }^{3}$ Jaseng Spine \& Joint Research Institute, Jaseng Medical Foundation, Gangnam-gu, Republic of Korea

${ }^{4}$ Acupuncture \& Moxibustion, Dongshin University College of Korean Medicine, Naju, Republic of Korea

${ }^{5}$ Department of Acupuncture \& Moxibustion, Kyung Hee University, Dongdaemungu, Seoul, Republic of Korea

${ }^{6}$ Jaseng Spine and Joint Research Institute, Jaseng Medical Foundation, Gangnamgu, Republic of Korea

Contributors $\mathrm{H}-\mathrm{sR}, \mathrm{BJ}$ and $\mathrm{I}-\mathrm{HH}$ contributed to the conceptualisation and investigation. $\mathrm{BJ}$ and $\mathrm{JY}$ conducted the data curation and formal analysis. BJ and $\mathrm{I}-\mathrm{HH}$ contributed to the methodology, project administration and supervision. J-HK and DN contributed to the validation.

Funding The authors have not declared a specific grant for this research from any funding agency in the public, commercial or not-for-profit sectors.

Competing interests None declared.

Patient consent for publication Not required.

Ethics approval The study was approved by the Institutional Review Board of Jaseng Hospital of Korean Medicine in Seoul, Korea (JASENG 2019-08-006).

Provenance and peer review Not commissioned; externally peer reviewed.

Data availability statement Data are available in a public, open access repository. The datasets generated during and/or analysed in the current study are available in the HIRA-NPS repository. The study used HIRA data (2015-2017), which are third-party data and thus not owned by the authors. The HIRA data are available on direct request, via email or fax, and submission of the request form and declaration of data use, which are downloadable from the HIRA website (http://opendata.hira.or. $\mathrm{kr}$ ), and on payment of a data request fee ( $300000 \mathrm{KRW}$ per dataset).

Open access This is an open access article distributed in accordance with the Creative Commons Attribution Non Commercial (CC BY-NC 4.0) license, which permits others to distribute, remix, adapt, build upon this work non-commercially, and license their derivative works on different terms, provided the original work is properly cited, appropriate credit is given, any changes made indicated, and the use is non-commercial. See: http://creativecommons.org/licenses/by-nc/4.0/.

ORCID iDs

Boyoung Jung http://orcid.org/0000-0001-9812-0745

In-Hyuk Ha http://orcid.org/0000-0002-5020-6723

\section{REFERENCES}

1 Papa JA. Clinical orthopaedic rehabilitation: an evidence-based Approach-Third edition. J Can Chiropr Assoc 2012;56:234.

2 O'Neill PJ, Parks BG, Walsh R, et al. Excursion and strain of the superficial peroneal nerve during inversion ankle sprain. J Bone Joint Surg Am 2007;89:979-86.

3 Waterman BR, Owens BD, Davey S, et al. The epidemiology of ankle sprains in the United States. J Bone Joint Surg Am 2010;92:2279-84.

4 National Health Insurance Service. National health insurance statistical Yearbook. National Health Insurance Service, 2018.

5 Knowles SB, Marshall SW, Miller T, et al. Cost of injuries from a prospective cohort study of North Carolina high school athletes. Inj Prev 2007;13:416-21.

6 Konradsen L, Bech L, Ehrenbjerg M, et al. Seven years followup after ankle inversion trauma. Scand J Med Sci Sports 2002;12:129-35.

7 Soboroff SH, Pappius EM, Komaroff AL. Benefits, risks, and costs of alternative approaches to the evaluation and treatment of severe ankle sprain. Clin Orthop Relat Res 1984;183:160-8.

8 Verhagen RA, de Keizer G, van Dijk CN. Long-term follow-up of inversion trauma of the ankle. Arch Orthop Trauma Surg 1995;114:92-6.

9 Jung B, Bae S, Kim S. Use of Western medicine and traditional Korean medicine for joint disorders: a retrospective comparative analysis based on Korean nationwide insurance data. Evid Based Complement Alternat Med 2017;2017:1-31.

10 National Development Institute of Korean Medicine.. NIKOM report of Korean medicine policy, 2016.

11 Jung B, Kim J, Ha I-H, et al. Factors affecting utilisation of traditional Korean medical services by privately insured persons: a retrospective study using Korean health panel survey (KHPS). BMJ Open 2020;10:e033159. 
12 Kannus P, Renstrom P. Treatment for acute tears of the lateral ligaments of the ankle. J Bone Joint Surg Am 1991;73:305-12.

13 Kerkhoffs GMMJ, Rowe BH, Assendelft WJJ, et al. Immobilisation and functional treatment for acute lateral ankle ligament injuries in adults. Cochrane Database Syst Rev 2002:CD003762.

14 Kerkhoffs GMMJ, Struijs PAA, Marti RK, et al. Different functional treatment strategies for acute lateral ankle ligament injuries in adults. Cochrane Database Syst Rev 2002:CD002938.

15 Pijnenburg AC, Van Dijk CN, Bossuyt PM, et al. Treatment of ruptures of the lateral ankle ligaments: a meta-analysis. J Bone Joint Surg Am 2000;82:761-73.

16 Sung KS, Park JM. Acute ankle sprains. Arthrosc Orthop Sports Med 2016;3:11-17.

17 Hootman JM, Dick R, Agel J. Epidemiology of collegiate injuries for 15 sports: summary and recommendations for injury prevention initiatives. J Athl Train 2007;42:311.

18 Hunt KJ, Lawson P. Acute ankle ligament injuries. In: Sports injuries of the foot and ankle. Springer, 2019: 3-12.

$19 \mathrm{Kim}$ JA, Yoon S, Kim LY, et al. Towards actualizing the value potential of Korea Health Insurance Review and Assessment (HIRA) data as a resource for health research: strengths, limitations, applications, and strategies for optimal use of HIRA data. J Korean Med Sci 2017;32:718-28.

$20 \mathrm{Kim}$ L, Kim J-A, Kim S. A guide for the utilization of health insurance review and assessment service national patient samples. Epidemiol Health 2014;36:e2014008.

21 Kim SK, Kleimeyer JP, Ahmed MA, et al. Two genetic loci associated with ankle injury. PLoS One 2017;12:e0185355.

22 Doherty C, Delahunt E, Caulfield B, et al. The incidence and prevalence of ankle sprain injury: a systematic review and metaanalysis of prospective epidemiological studies. Sports Med 2014;44:123-40.

23 Kwon S. Health care financing and delivery for the poor in Korea. International Rev Public Admin 2000;5:37-45.

24 Kerkhoffs GMMJ, Handoll HHG, de Bie R, et al. Surgical versus conservative treatment for acute injuries of the lateral ligament complex of the ankle in adults. Cochrane Database Syst Rev 2007:CD000380.
25 Peters JW, Trevino SG, Renstrom PA. Chronic lateral ankle instability. Foot Ankle 1991;12:182-91.

26 Lee JY, Jo M-W, Yoo W-S, et al. Evidence of a broken healthcare delivery system in Korea: unnecessary Hospital outpatient utilization among patients with a single chronic disease without complications. J Korean Med Sci 2014;29:1590-6.

27 Park JY, Chai H-Y. The effects of an increase of outpatient Coinsurance rate on the financial status of the National health insurance. Korean Insurance J 2003;64:109-36.

28 Miklovic TM, Donovan L, Protzuk OA, et al. Acute lateral ankle sprain to chronic ankle instability: a pathway of dysfunction. Phys Sportsmed 2018;46:116-22.

29 Aronson JK. Meyler's side effects of analgesics and anti-inflammatory drugs. Elsevier, 2009.

30 Buchman AL. Side effects of corticosteroid therapy. J Clin Gastroenterol 2001;33:289-94.

31 National Health Insurance Service. Claim status for covered drugs. National Health Insurance Service, 2017.

32 Brison RJ, Day AG, Pelland L, et al. Effect of early supervised physiotherapy on recovery from acute ankle sprain: randomised controlled trial. BMJ 2016;355:i5650.

$33 \mathrm{Lim} \mathrm{J}-\mathrm{H}$, Lim S-M. A literature study about utilization of Oriental medical services. J Korea Institute Orient Med Diagnos 2012;16:11-22.

34 Hwang J-set al. Study of the effect of blood-letting cupping treatment for acute ankle sprain: a randomize controlled trial. $J$ Acupuncture Res 2005;22:243-52.

35 Park YS, Kim JS. Analysis of the current status outpatient utilization of medical and Korean traditional medical institutions: focuse on outpatient frequent outpatient diseases. J Korean Med Assoc 2017;60:912-9.

36 Park B. Studying on diagnosis accuracy for health insurance claims data in Korea. Seoul: Seoul National University, 2003: 17-29.

37 Hodgson TA, Meiners MR. Cost-Of-IIIness methodology: a guide to current practices and procedures. Milbank Mem Fund Q Health Soc 1982;60:429-62. 